Pensamiento Crítico Vol. 19 № 2, pp. 167-176

\title{
Costos por actividad
}

\author{
The activity-based cost
}

Raimundo Renaun Pacheco Mexzon*

\section{RESUMEN}

Cuando una empresa produce para el mercado más de un producto, debe asignar los costos indirectos de fabricación (CIF) de la mejor manera posible a estos productos. El sistema de costos por actividad, llamado también sistema $A B C$, permite que los $\mathrm{CIF}$ se apliquen a cada producto de manera más real. Por lo tanto, es objetivo del presente artículo detallar cómo aplicar este sistema y comparar el resultado con el método tradicional.

Palabras clave: Costos por actividad, generador de costos, costos indirectos de fabricación.

\begin{abstract}
When a company produces for the market more than one product should allocate Indirect Costs Manufacturing (ICM) in the best possible way to these products. The activity-based cost system, also called ABC system allows each product ICM more realistically apply. Therefore it is an object of this article how to apply this system and compare the result with the traditional method.
\end{abstract}

Keywords: Activity costs, generator costs, indirect cost manufacturing.

* Ingeniero economista por la Universidad Nacional de Ingeniería. Magíster en Economía con mención en Finanzas y doctor en Economía por la Universidad Nacional Mayor de San Marcos. 


\section{Pensamiento Crítico Vol. 19. № 2}

\section{Costo de fabricación o costo de producción}

Una empresa para producir bienes o servicios que saca al mercado combina los factores productivos mano de obra, materiales o insumos y capital reflejado en maquinaria y equipo.

En el proceso productivo la mano de obra puede ser clasificada como mano de obra directa y mano de obra indirecta. Los materiales o insumos también siguen el mismo principio, es decir pueden ser materiales directos o materiales indirectos. Los otros costos y gasto de fabricación, que no son mano de obra ni materiales, se les consideran como otros costos o gastos indirectos de fabricación.

Por lo tanto, el costo de fabricación o producción de bienes o servicios que produce una empresa queda resumido en: mano de obra directa (MOD) más materiales directos (MATD) más costos indirectos de fabricación (CIF).

Por ejemplo, si la mano de obra directa para producir los bienes o servicios es $\$ 200,000$. Los materiales directos llegan a $\$ 500,000$. Y los costos y gastos indirectos de fabricación llegan a $\$ 300,000$, dentro de los cuales la mano de obra indirecta es $\$$ 50,000 , los materiales indirectos $\$ 90,000$ y los otros costos indirectos $\$ 160,000$, el costo de fabricación o producción se presentaría en resumen de la siguiente manera:

Costo de fabricación o producción

\begin{tabular}{|l|c|}
\hline \multicolumn{1}{|c|}{ RUBROS } & MONTO EN \$ \\
\hline MOD & 200,000 \\
\hline MATD & 500,000 \\
\hline CIF & 300,000 \\
\hline Mano de obra indirecta (MOind) & 50,000 \\
\hline Materiales indirectos (MATind) & 90,000 \\
\hline Otros costos indirectos (OCind) & 160,00 \\
\hline Total costo de fabricación o costo de producción & \multicolumn{1}{|c|}{$\mathbf{1}^{\prime} \mathbf{0 0 0 , 0 0 0}$} \\
\hline
\end{tabular}

Como se puede apreciar en este caso, el CIF de la empresa llega a $\$ 300,000$ y si la empresa solo produce un bien o un servicio, los $\$ 300,000$ serían absorbidos por este 


\section{Raimundo Renaun Pacheco Mexzon}

producto o servicio. Pero si la empresa produce más de un producto o servicio, debería asignarse parte de este costo indirecto de fabricación a cada producto o servicio.

Para asignar los CIF a cada producto o servicio se tienen dos métodos: el método tradicional y el método de costos por actividad o ABC.

Según los expertos, el sistema de costos por actividad o ABC permite asignar los costos indirectos de fabricación de manera más real que el método tradicional.

\section{Costos por actividad o sistema de costos $A B C$}

Es un sistema de costos en el que los costos indirectos de fabricación (CIF) se aplican primero a las actividades indirectas necesarias para la fabricación de los productos y luego, se calculan y asignan por cada producto.

Se considera que con este sistema los costos indirectos de fabricación (CIF) quedan asignados a los productos de manera más ajustada a la realidad.

Para entender la metodología de cálculo, desarrollaremos el siguiente ejemplo:

\section{Ejemplo de aplicación}

Una empresa tiene en costos indirectos de fabricación (CIF) US\$2'000,000. Está produciendo dos productos: Uno de lujo y uno regular. Utiliza los recursos que se indican en el cuadro siguiente:

\begin{tabular}{|l|c|c|}
\hline \multicolumn{1}{|c|}{ RUBROS } & PRODUCTO de LUJO & PRODUCTO REGULAR \\
\hline Materiales Directos por Unidad (MATD) & $\$ 150$ & $\$ 112$ \\
\hline Mano de Obra Directa por Unidad (MOD) & $\$ 16$ & $\$ 8$ \\
\hline Horas de MOD por Unidad & 1.6 & 0.8 \\
\hline Volumen de Producción & 5,000 Unidades & 40,000 Unidades \\
\hline
\end{tabular}

Para la producción de estos dos productos (producto de lujo y producto regular) la empresa tiene costos indirectos de fabricación (CIF) identificados por actividades tales como: 


\section{Pensamiento Crítico Vol. 19. № 2}

- Abastecimiento.

- Reprocesamiento.

- Control de calidad.

- Utilización de máquinas.

El detalle de los costos indirectos de fabricación (CIF) por actividades se muestra a continuación:

\begin{tabular}{|c|c|c|c|c|c|}
\hline \multirow[t]{2}{*}{ ACTIVIDADES } & \multirow[t]{2}{*}{$\begin{array}{c}\text { Generador de costo } \\
\text { o unidad de medida } \\
\text { de la actividad }\end{array}$} & \multirow[t]{2}{*}{$\begin{array}{c}\text { Costo } \\
\text { indirecto por } \\
\text { actividad }\end{array}$} & \multicolumn{3}{|c|}{$\begin{array}{l}\text { Unidades de actividad por } \\
\text { unidad de producto }\end{array}$} \\
\hline & & & De lujo & Regular & TOTAL \\
\hline Abastecimiento & Ordenes & $\$ 84,000$ & 400 & 800 & 1200 \\
\hline Reprocesamiento & Ordenes & $\$ 216,000$ & 300 & 600 & 900 \\
\hline Control de calidad & Tareas & $\$ 450,000$ & 4,000 & 11,000 & 15,000 \\
\hline Utilización de máquinas & Horas máquina & $\$ 1^{\prime} 250,000$ & 20,000 & 30,000 & 50,000 \\
\hline TOTAL CIF & & $\$ 2 ’ 000,000$ & & & \\
\hline
\end{tabular}

Como se puede observar en este cuadro resumen, los generadores de costos o unidades en que se miden las actividades nos indican que la actividad de abastecimiento y reprogramación se genera en órdenes, el control de calidad en tareas y la utilización de máquinas en horas. Cada una de estas actividades que conforman los costos indirectos de fabricación de esta empresa demandan mano de obra indirecta, materiales indirectos y otros costos indirectos que en total suman los $\$ 2 ' 000,000$ de costo indirecto de fabricación (CIF) de la empresa.

También se aprecia que por cada actividad hay un costo indirecto de fabricación que suman los $\$ 2^{\prime} 000,000$. Así para la actividad de abastecimiento el CIF es $\$ 84,000$. Para la actividad de reprocesamiento es \$216,000. Para la actividad de control de calidad es $\$ 450,000$ y para la actividad de utilización de maquinaria es \$1'250,000.

Se muestra también en este cuadro la utilización de una cantidad de unidades de generadores de costos por actividad y por cada unidad de producto (producto de lujo y producto regular). Así la actividad de abastecimiento utiliza 1,200 órdenes de las 


\section{Raimundo Renaun Pacheco Mexzon}

cuales 400 corresponden al producto de lujo y 800 al producto regular. La actividad de reprocesamiento utiliza 900 órdenes, de las cuales 300 son para el producto de lujo y 600 para el producto regular. La actividad de control de calidad utiliza 15,000 tareas, de las cuales 4,000 son para el producto de lujo y 11,000 para el producto regular. Por último, la actividad de máquinas consume 50,000 horas máquina, de las cuales 20,000 corresponden al producto de lujo y 30,000 al producto regular.

Para asignar los CIF a cada producto, tenemos que calcular en primer lugar las tasas que relacionan el costo indirecto de fabricación de cada actividad con el generador de costos que son las unidades de medida de la actividad.

\section{Cálculo de la tasa de costo indirecto por actividad}

\section{Tasa $=$ CIF por actividad $\div$ Total unidades de actividad}

Para abastecimiento $=\$ 84,000 \div 1200$ órdenes $=\$ 70$ por orden .

Para reprocesamiento $=\$ 216,000 \div 900$ órdenes $=\$ 240$ por orden.

Para control de calidad $=\$ 450,000 \div 15,000$ tareas $=\$ 30$ por tarea .

Para máquinas $=\$ 1 ’ 250,000 \div 50,000$ horas máquina $=\$ 25$ por hora máquina.

Luego multiplicamos esta tasa por el número de unidades de medida de la actividad para cada producto. Ejemplo, para abastecimiento: $\$ 70$ x $400=\$ 28,000$. Para las otras actividades se procede igual. Ver cuadro siguiente.

Cálculo del CIF asignado a cada producto

\begin{tabular}{|l|c|c|c|c|c|}
\hline \multicolumn{1}{|c|}{ ACTIVIDAD } & TASA & $\begin{array}{c}\text { Unidades de } \\
\text { actividad por } \\
\text { producto de lujo }\end{array}$ & $\begin{array}{c}\text { Costo asignado } \\
\text { a unidades de } \\
\text { producto de lujo }\end{array}$ & $\begin{array}{c}\text { Unidades de } \\
\text { actividad por } \\
\text { producto regular }\end{array}$ & $\begin{array}{c}\text { Costo asignado } \\
\text { a unidades de } \\
\text { producto regular }\end{array}$ \\
\hline Abastecimiento & $\$ 70$ & 400 & $\mathbf{\$ 2 8 , 0 0 0}$ & 800 & $\mathbf{5 6 , 0 0 0}$ \\
\hline Reprocesamiento & $\$ 240$ & 300 & $\mathbf{\$ 7 2 , 0 0 0}$ & 600 & $\mathbf{1 4 4 , 0 0 0}$ \\
\hline Control de calidad & $\$ 30$ & 4,000 & $\mathbf{\$ 1 2 0 , 0 0 0}$ & 11,000 & $\mathbf{3 3 0 , 0 0 0}$ \\
\hline Utilización de máquinas & $\$ 25$ & 20,000 & $\mathbf{\$ 5 0 0 , 0 0 0}$ & 30,000 & $\mathbf{7 5 0 , 0 0 0}$ \\
\hline TOTAL CIF & & & $\mathbf{\$ 7 2 0 , 0 0 0}$ & & $\mathbf{\$ 1} \mathbf{2 8 0 , 0 0 0}$ \\
\hline
\end{tabular}




\section{Pensamiento Crítico Vol. 19. N²}

De acuerdo al cuadro anterior se aprecia que para el producto de lujo los costos indirectos de fabricación llegarían a $\$ 720,000$ y para el producto regular a $\$$ $1^{\prime} 280,000$. La suma de ambos dan los $\$ 2^{\prime} 000,000$ que es el total de costos indirectos de fabricación de la empresa.

Como sabemos que la empresa fabrica 5,000 unidades del producto de lujo y 40,000 del producto regular podemos calcular el costo indirecto de fabricación por unidad de cada producto.

Cálculo del costo indirecto de fabricación (CIF) por unidad de producto:

Producto de lujo: $\$ 720,000 \div 5,000=\$ 144$

Producto regular: $\$ 1 ' 280,000 \div 40,000=\$ 32$

También podemos calcular el costo unitario para cada producto sumando los materiales directos, la mano de obra directa y el costo indirecto de fabricación por unidad.

\section{Costo unitario por producto}

\begin{tabular}{|l|c|c|}
\hline \multicolumn{1}{|c|}{ RUBROS } & PRODUCTO DE LUJO & PRODUCTO REGULAR \\
\hline Materiales directos por unidad & $\$ 150$ & $\$ 112$ \\
\hline Mano de obra directa por unidad & $\$ 16$ & $\$ 8$ \\
\hline Costo indirecto de fabricación por unidad & $\$ 144$ & $\$ 32$ \\
\hline Costo unitario & $\mathbf{\$ 3 1 0}$ & $\mathbf{\$ 1 5 0}$ \\
\hline
\end{tabular}

\section{Método tradicional}

Los costos indirectos de fabricación también se pueden asignar a cada producto utilizando el método tradicional. En este método se toma como base para la asignación el número de horas hombre de la mano de obra directa. 


\section{Raimundo Renaun Pacheco Mexzon}

La tasa de asignación del costo indirecto de fabricación en relación con la mano de obra directa se calcula dividiendo el costo indirecto de fabricación entre el total de horas hombre de mano de obra directa. La fórmula que se utiliza se presenta a continuación:

$$
\text { Tasa }=\text { CIF } \div \text { Total horas de MOD }
$$

Tomando en consideración la información de la empresa, se procede a calcular el total de horas de mano de obra directa, conforme se indica en el cuadro siguiente. El total es de 40,000 horas de MOD.

Total de horas de MOD

\begin{tabular}{|l|c|c|c|}
\hline \multicolumn{1}{|c|}{ RUBROS } & PRODUCTO de LUJO & PRODUCTO REGULAR & TOTAL \\
\hline Horas de MOD por unidad & 1.6 & 0.8 & \\
\hline Volumen de producción & 5,000 Unidades & 40,000 Unidades & \\
\hline TOTAL horas de MOD & $\mathbf{1 . 6} \times \mathbf{5 , 0 0 0}=\mathbf{8 , 0 0 0}$ & $\mathbf{0 . 8} \times \mathbf{4 0 , 0 0 0}=\mathbf{3 2 , 0 0 0}$ & $\mathbf{4 0 , 0 0 0}$ \\
\hline
\end{tabular}

Cálculo de la tasa de asignación del costo indirecto a la MOD

$$
\begin{gathered}
\text { Tasa }=\text { CIF } \div \text { Total horas de MOD } \\
\text { Tasa }=\$ 2 ' 000,000 / 40,000 \text { horas de MOD } \\
\text { TASA }=\$ 50 \text { por hora de MOD } \\
\hline
\end{gathered}
$$

Como se conoce para cada producto las horas de mano de obra directa se puede calcular el costo indirecto de fabricación multiplicando la tasa por las horas de mano de obra directa de cada producto. En el cuadro siguiente se presenta este cálculo: 
Pensamiento Crítico Vol. 19. № 2

\section{Costo indirecto de fabricación para cada producto}

\begin{tabular}{|l|c|c|}
\hline \multicolumn{1}{|c|}{ RUBROS } & PRODUCTO DE LUJO & PRODUCTO REGULAR \\
\hline Horas de mano de obra directa por unidad & 1.6 & 0.8 \\
\hline Tasa de CIF por unidad de mano de obra directa & $\$ 50$ & $\$ 50$ \\
\hline CIF por unidad & $\mathbf{1 . 6} \times \mathbf{5 0}=\mathbf{\$ 8 0}$ & $\mathbf{0 . 8} \times \mathbf{5 0}=\mathbf{\$ 4 0}$ \\
\hline
\end{tabular}

Se aprecia que el costo indirecto de fabricación para el producto de lujo es $\$ 80$ por unidad producida y de $\$ 40$ para el producto regular.

Como se conoce el costo de la mano de obra directa, el costo de los materiales directos y los costos indirectos por unidad producida podemos calcular el costo unitario para cada producto. En el cuadro siguiente se presenta este cálculo:

\section{Costo unitario para cada producto}

\begin{tabular}{|l|c|c|}
\hline \multicolumn{1}{|c|}{ RUBROS } & PRODUCTO de LUJO & PRODUCTO REGULAR \\
\hline Materiales directos por unidad & $\$ 150$ & $\$ 112$ \\
\hline Mano de obra directa por unidad & $\$ 16$ & $\$ 8$ \\
\hline CIF por unidad & $\$ 80$ & $\$ 40$ \\
\hline Costo unitario & $\mathbf{\$ 2 4 6}$ & $\mathbf{\$ 1 6 0}$ \\
\hline
\end{tabular}

Como se puede apreciar, con el método tradicional el costo unitario para el producto de lujo es $\$ 246$ y para el producto regular $\$ 160$.

\section{Comparación de ambos métodos}

Al comparar los resultados de ambos métodos, se aprecia un ajuste en la asignación del costo indirecto de fabricación (CIF).

Con el método tradicional, el producto de lujo tiene un costo indirecto de fabricación unitario de $\$ 80$, mientras que con el método de costos por actividad o $\mathrm{ABC}$ es de $\$ 144$, es decir se incrementa. 


\section{Raimundo Renaun Pacheco Mexzon}

Por otro lado, con el método tradicional el producto regular tiene un costo indirecto de fabricación unitario de $\$ 40$ mientras que con el método de costos por actividad o $\mathrm{ABC}$ es de $\$ 32$. Es decir, disminuye.

Este resultado afecta también los costos unitarios totales de cada producto. Así, el producto de lujo con el método tradicional tiene un costo unitario total de $\$ 246$ y el producto regular $\$ 160$. Mientras que con el método de costos por actividad o $\mathrm{ABC}$ el producto de lujo tiene un costo total unitario de $\$ 310$ y el producto regular $\$ 150$.

En el cuadro siguiente se resume lo mencionado anteriormente:

\begin{tabular}{|l|c|c|c|c|}
\hline & \multicolumn{2}{|c|}{ MÉTODO TRADICIONAL } & \multicolumn{2}{c|}{ COSTO POR ACTIVIDAD } \\
\hline \multicolumn{1}{|c|}{ RUBROS } & $\begin{array}{c}\text { PRODUCTO } \\
\text { DE LUJO }\end{array}$ & $\begin{array}{c}\text { PRODUCTO } \\
\text { REGULAR }\end{array}$ & $\begin{array}{c}\text { PRODUCTO } \\
\text { DE LUJO }\end{array}$ & $\begin{array}{c}\text { PRODUCTO } \\
\text { REGULAR }\end{array}$ \\
\hline Mat. directos por unidad & $\$ 150$ & $\$ 112$ & $\$ 150$ & $\$ 112$ \\
\hline MOD por unidad & $\$ 16$ & $\$ 8$ & $\$ 16$ & $\$ 8$ \\
\hline CIF por unidad & $\$ 80$ & $\$ 40$ & $\$ 144$ & $\$ 32$ \\
\hline Costo unitario & $\mathbf{\$ 2 4 6}$ & $\mathbf{\$ 1 6 0}$ & $\mathbf{\$ 3 1 0}$ & $\mathbf{\$ 1 5 0}$ \\
\hline
\end{tabular}

Por esta diferencia que se genera al utilizar uno u otro método de asignación, es que los expertos mencionan que con el método de costeo por actividad o método $\mathrm{ABC}$ se asignan los costos indirectos de fabricación de una manera más acorde con la realidad.

\section{En resumen:}

Al comparar los resultados de ambos métodos se aprecia un ajuste en la asignación del CIF. Para el producto de lujo se incrementa el costo indirecto unitario de $\$ 80$ a $\$ 144$, mientras que para el producto regular disminuye de $\$ 40$ a $\$ 32$.

El costo unitario de cada producto también se modifica pasando de $\$ 246$ a $\$ 310$ para el producto de lujo y de $\$ 160$ a $\$ 150$ para el producto regular 


\section{Pensamiento Crítico Vol. 19. No 2}

\section{Conclusiones}

1. Para que el gerente financiero pueda tener una buena gestión de los costos de la empresa debe conocer conceptualmente la diferencia entre el método tradicional y el método del costeo por actividad o ABC para asignar mejor los costos indirectos de fabricación.

2. El método de costos por actividad o $\mathrm{ABC}$ permite asignar de una forma más cercana a la realidad los costos indirectos de fabricación de la empresa.

3. Al comparar ambos métodos se aprecia la diferencia que se genera en el costo unitario de cada producto que fabrica la empresa.

\section{Recomendaciones}

1. Divulgar el concepto del costeo por actividad en todos los ejecutivos de la empresa para reforzar la idea de que los costos indirectos de fabricación deben ser asignados más acordes a la realidad en que opera la empresa.

2. Determinar periódicamente las actividades que deben tenerse en cuenta para asignar de la mejor manera posible los costos indirectos de fabricación a los bienes y servicios que produce y vende la empresa.

\section{Referencias bibliográficas}

Hansen Don R., Mowen Maryanne M. (2008). Administración de costos. Editorial Thomson.

Bendersky, Eduardo (2011). ABC-ABM Gestión de costos por actividades. Ediciones Priale.

Rodríguez, Martín; Ramón, Alejandro (2007). Modelos de coste ABC. Ediciones Diccionario Económico. 